\title{
Transport and disruption of Bose-Einstein condensates in optical lattices
}

\author{
R. G. Scott, A. M. Martin, S. Bujkiewicz, and T. M. Fromhold \\ School of Physics and Astronomy, University of Nottingham, Nottingham NG7 2RD, United Kingdom
}

N. Malossi, O. Morsch, M. Cristiani, and E. Arimondo

INFM, Dipartimento di Fisicia, Università di Pisa, Via Buonarroti 2, I-56127 Pisa, Italy

(Received 26 September 2003; published 16 March 2004)

\begin{abstract}
We present theoretical and experimental studies of the dynamics of Bose-Einstein condensates in a onedimensional optical lattice and a three-dimensional harmonic trap. For low atom densities and inertial forces, the condensate performs regular Bloch oscillations, and its center-of-mass motion closely follows semiclassical single-particle trajectories, shaped by the lowest-energy band. But in other regimes, the center-of-mass motion disrupts the internal structure of the condensate by generating solitons and vortex rings, which can trigger explosive expansion of the atom cloud. We use images of the atom cloud to provide experimental evidence for this internal disruption, and find that the process occurs most readily in high-density condensates undergoing slow Bloch oscillations.
\end{abstract}

DOI: 10.1103/PhysRevA.69.033605

PACS number(s): 03.75.Kk, 05.45.Yv, 32.80.Pj, 42.50.Vk

\section{INTRODUCTION}

Optical lattices are currently attracting considerable theoretical and experimental interest, in part because they provide an opportunity to investigate the predictions of solidstate physics, but with a far greater degree of experimental control than is possible with crystalline periodic potentials. Consequently, optical lattices have been used to observe atom diffraction [1], Bloch oscillations [2], and WannierStark ladders [3]. As a natural progression from studies of noninteracting ultracold atom clouds in optical lattices, recent work has focused on the dynamics of Bose-Einstein condensates in optical lattices [4-24]. Initially, this work investigated effects similar to those observed previously for noninteracting ultracold atom clouds. For example, BoseEinstein condensates were observed to undergo Bloch oscillations $[4,5]$ and diffraction [6,7]. However, further work revealed that the interatomic interactions, described by the nonlinear term in the Gross-Pitaevskii equation, can have a pronounced effect on the transport properties of condensates in optical lattices, and produce completely different dynamics from those observed for individual atoms. There has been particular interest in the instability and disruption of condensates in optical lattices [8-11], and in the breakdown of Bloch oscillations $[4,5,9,12]$. Such effects have been attributed to Landau-Zener tunneling $[4,5,11,13]$, dynamical instability [11], and Landau instability [8].

In previous work [25], we demonstrated that Bragg reflection of a Bose-Einstein condensate can generate solitons and vortices, hence disrupting the internal structure of the atom cloud and damping the Bloch oscillations. Furthermore, we showed that the density of the condensate is a crucial factor in determining the extent of the disruption. In this paper, we study the dynamics of Bose-Einstein condensates in optical lattices in more detail, by considering two systems realized in recent experiments $[4,8]$. In particular, we investigate how the temporal period of the Bloch oscillations affects soliton and vortex production. We show that solitons and vortices form more readily for low-frequency Bloch oscillations, whose period is much longer than the condensate correlation time, that is, the time taken for the atom density profile to respond to a perturbation. Our results provide insights for understanding the mechanisms of soliton and vortex production, and identify key factors that determine the dynamical behavior of condensates in optical lattices. Moreover, we present experimental data and corresponding theoretical simulations, which provide direct evidence for our previous prediction that Bragg reflection can severely disrupt the atom cloud [25]. Our calculations are in good quantitative agreement with the experimental data presented in this paper, and also with previously published experimental work [8].

The two systems, referred to as $A$ and $B$, are analyzed in Secs. III and IV, respectively. System $A$ was realized in recent experiments by Morsch et al. [4], which demonstrated Bloch oscillations of Bose-Einstein condensates in an accelerating optical lattice. We simulate these Bloch oscillations by solving the three-dimensional Gross-Pitaevskii equation. Furthermore, by adjusting the system parameters we identify a regime of condensate behavior, in which solitons and vortices disrupt the internal structure of the atom cloud. The theoretical predictions are related to experimental data that show the disruption of the condensate as it reaches the Brillouin-zone boundary. System $B$ was realized in recent experiments by Burger and co-workers [8]. These experiments concerned the motion of a Bose-Einstein condensate in an optical lattice, and revealed, under certain conditions, a distortion and broadening of the condensate, and damping of the condensate oscillations. Burger and co-workers explained their results in terms of a Landau instability of the condensate. Landau instability occurs when the condensate velocity exceeds the local speed of sound, causing production of elementary excitations and heating of the atom cloud. This interpretation was questioned by $\mathrm{Wu}$ and $\mathrm{Niu}[26,27]$, who accounted for the distortion of the condensate as mainly due to a dynamical instability. However, their work does not describe the subsequent effects of the dynamical instability on the condensate motion or internal structure. The results in Sec. IV provide a complementary interpretation, in which the 
instability of the condensate originates from rapid vortex production at the point of Bragg reflection, leading to disruption and explosive expansion of the atom cloud.

\section{THEORETICAL MODEL OF A BOSE-EINSTEIN CONDENSATE IN A ONE-DIMENSIONAL OPTICAL LATTICE AND A THREE-DIMENSIONAL HARMONIC TRAP}

We consider ${ }^{87} \mathrm{Rb}$ Bose-Einstein condensates containing $N$ atoms in a one-dimensional optical lattice, formed by two counterpropagating laser beams of wavelength $\lambda$, and a three-dimensional harmonic trap. The potential energy of a single atom in the optical lattice is

$$
V_{\mathrm{OL}}(x)=V_{0} \sin ^{2}(\pi x / d),
$$

where $V_{0}$ and $d=\lambda / 2$ are, respectively, the depth and period of the optical potential. The harmonic trap is symmetrical under rotation about the axis of the optical lattice (the $x$ axis). Its potential-energy profile is given by

$$
V_{\text {trap }}(x, r)=\frac{m}{2}\left(\omega_{x}^{2} x^{2}+\omega_{r}^{2} r^{2}\right),
$$

where $m$ is the mass of a single ${ }^{87} \mathrm{Rb}$ atom, $\omega_{x}$ and $\omega_{r}$ are the trap angular frequencies, and

$$
r=\sqrt{y^{2}+z^{2}}
$$

is the radial coordinate.

Due to its translational symmetry, the optical lattice generates an energy band structure for motion in the $x$ direction. We calculated the energy-wave-vector dispersion relation for the two lowest-energy bands using Mathieu functions [28]. In principle, the harmonic trap breaks the translational symmetry of the potential. However, for the parameters considered in this paper, the condensate remains sufficiently close to the trap center to ensure that the change in $V_{\text {trap }}(x, r)$ across each lattice period is much less than the width of the lowest-energy band. Consequently, the trap potential can be treated as a perturbation to the lattice potential, and the band structure remains intact $[25,29]$. Moreover, in the following sections, we show that the band structure plays a crucial role in the condensate dynamics.

The total potential energy of a single atom in the optical lattice and harmonic trap is

$$
V_{\mathrm{T}}(x, r)=V_{\mathrm{OL}}(x)+V_{\text {trap }}(x, r) .
$$

Since $V_{\mathrm{T}}(x, r)$ is invariant under rotation about the $x$ axis, the condensate wave function is described in cylindrical polar coordinates $(x, r, \varphi)$, and is taken to be of the form

$$
\Psi(x, r, \varphi, t)=\psi(x, r, t) e^{i l \varphi},
$$

where

$$
\varphi=\tan ^{-1}\left(\frac{z}{y}\right)
$$

and $l=0, \pm 1, \pm 2, \ldots$ is the quantum number for the $x$ component of angular momentum $l \hbar$. Here, we consider the con- densate ground state with $l=0$, which, as in the experiments of Refs. [4,8], does not rotate about the $x$ axis. Consequently, any derivatives of $\Psi$ with respect to $\varphi$ are zero, and we can determine the condensate dynamics by solving the effective two-dimensional time-dependent Gross-Pitaevskii equation

$i \hbar \frac{\partial \Psi(x, r, t)}{\partial t}=\left[-\frac{\hbar^{2}}{2 m} \nabla^{2}+V_{\mathrm{T}}(x, r)+U_{0}|\Psi(x, r, t)|^{2}\right] \Psi(x, r, t)$,

where

$$
\nabla^{2}=\frac{\partial^{2}}{\partial x^{2}}+\frac{\partial^{2}}{\partial r^{2}}+\frac{1}{r} \frac{\partial}{\partial r}
$$

and

$$
U_{0}=\frac{4 \pi \hbar^{2} a}{m},
$$

in which $a=5.4 \mathrm{~nm}$ is the $s$-wave scattering length [30]. In our calculations, we express Eq. (7) as a finite-difference equation, and then solve it numerically using the CrankNicolson method, incorporating time or operator splitting [31].

\section{DYNAMICS OF SYSTEM $A$}

The parameters of system $A$ are $N=10^{4}, V_{0}=36.2 \mathrm{peV}$ $=2.32 E_{R}$ (where $E_{R}=h^{2} / 2 m \lambda^{2}$ is the photon recoil energy), $d=390 \mathrm{~nm}, \omega_{x}=2 \pi \times 42 \mathrm{rad} \mathrm{s}^{-1}$, and $\omega_{r}=2 \pi \times 25 \mathrm{rad} \mathrm{s}^{-1}$. Figure 1(a) shows the potential-energy profiles of the optical lattice (solid curve) and harmonic trap (dotted curve) along $r=0$. The energy range of the lowest band is indicated by the gray rectangle. The width of this band is $8.5 \mathrm{peV}=0.54 E_{R}$. The bottom of the second band is at $83 \mathrm{peV}=5.3 E_{R}$, which is well above the top of the optical potential. As a consequence, interband Zener tunneling is not important in this system. The equilibrium atom density profile of the condensate along $r=0$ is shown in Fig. 1(b). The density is strongly modulated by the optical lattice potential and has a peak value of $\sim 3.1 \times 10^{13} \mathrm{~cm}^{-3}$.

The condensate is set in motion by accelerating the optical potential. Experimentally [4], this was done by setting a constant frequency drift between the two laser beams, resulting in a constant acceleration of the optical lattice. In the absence of a magnetic trap, this acceleration is equivalent to the application of a constant inertial force on the atoms, which therefore perform Bloch oscillations in the rest frame of the lattice. The presence of the magnetic trap complicates the motion, since the accelerating optical lattice tends to drag the condensate up one side of the harmonic trap potential. Hence, the variation of the trap potential across each lattice period increases with time. As a consequence, the magnetic trap eventually begins to disrupt the band structure, which ultimately leads to the breakdown of Bloch oscillations.

Initially, we considered a lattice acceleration $\bar{a}=$ $-5.0 \mathrm{~ms}^{-2}$. We simulated the condensate motion by solving Eq. (7), and hence calculated the mean position of the condensate, $\langle x\rangle$, as a function of time $t$. We then determined the 

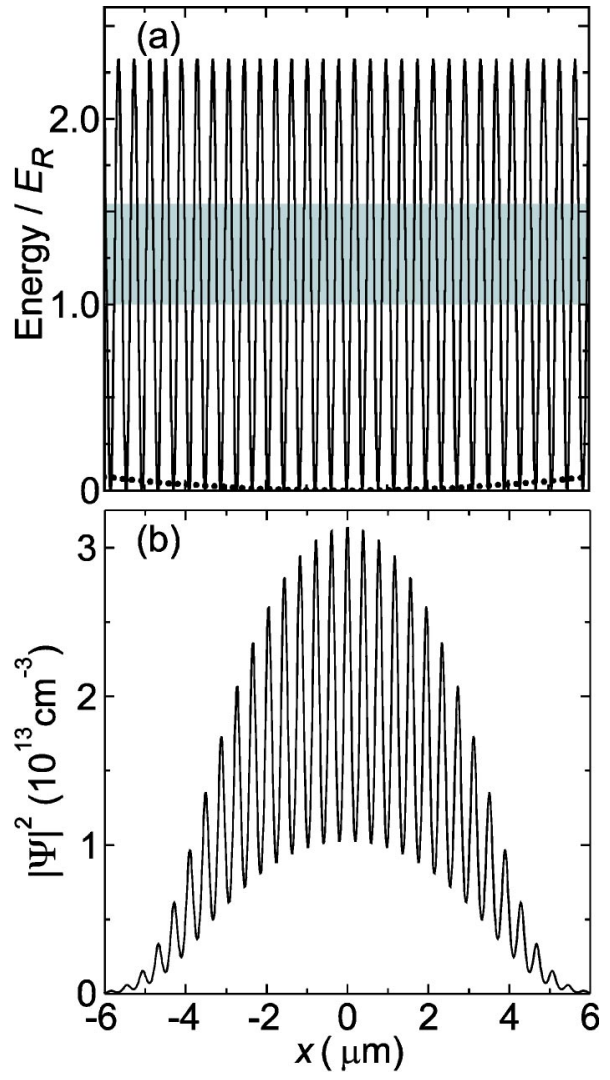

FIG. 1. (a) Solid curve: potential-energy profile of the optical lattice in system $A$. Gray rectangle: energy range of the lowestenergy band. Dotted curve: $x$ dependence of the harmonic potential energy along $r=0$. (b) Initial atom density profile of the condensate in system $A$, along $r=0$.

time evolution of this mean position in the rest frame of the optical lattice, $\langle x\rangle-x_{\mathrm{OL}}$ [solid curve in Fig. 2(a)], where

$$
x_{\mathrm{OL}}=\frac{1}{2} \bar{a} t^{2}
$$

is the lattice displacement resulting from the lattice acceleration $\bar{a}$, switched on abruptly at time $t=0$. Figure 2(a) shows that the condensate performs oscillations in the rest frame of the accelerating optical potential. Note that the amplitude and period of these oscillations decrease as $t$ increases, for a reason that we will explain later.

The condensate dynamics can also be represented in reciprocal space by taking the Fourier transform

$$
f\left(k_{x}, t\right)=\int_{-\infty}^{\infty} \psi(x, 0, t) e^{-i m \bar{a} t x / \hbar} e^{i k x} d x
$$

of the wave function along $r=0$. Note that the factor $e^{-i m a \bar{t} x / \hbar}$ is included in the integral in order to account for the acceleration of the optical lattice.

Figure 2(b) shows that the distribution of the Fourier power $\left|f\left(k_{x}, t\right)\right|^{2}$, corresponding to wave vector $k_{x}$, remains narrow throughout the simulation. The condensate's mean $k_{x}$ value increases approximately linearly with $t$ until it reaches the Brillouin-zone boundary, at which point the condensate undergoes Bragg reflection. Each Bragg reflection corre-

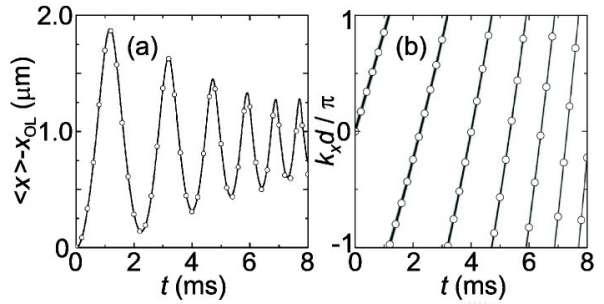

(c)

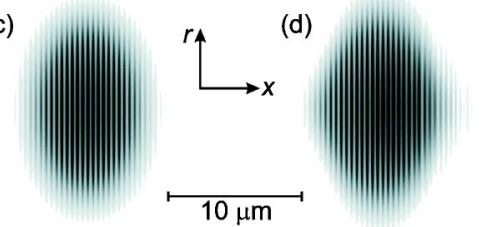

FIG. 2. (a) Solid curve: $\langle x\rangle-x_{\mathrm{OL}}$ vs $t$ calculated for system $A$ with $\bar{a}=-5.0 \mathrm{~ms}^{-2}$. Open circles: corresponding semiclassical trajectory. (b) Gray-scale plot: $\left|f\left(k_{x}, t\right)\right|^{2}$ within the first Brillouin zone (white $=0$, black high) for system $A$ with $\bar{a}=-5.0 \mathrm{~ms}^{-2}$. Open circles: points on corresponding semi-classical trajectory. (c) and (d) Gray-scale plots of condensate density (white $=0$, black high) in the $x-r$ plane (axes inset) for $t=0 \mathrm{~ms}$ and $8 \mathrm{~ms}$, respectively. Plots are symmetrical under rotation about the $x$ axis. Horizontal bar shows scale.

sponds to an upper turning point in Fig. 2(a). The lower turning points occur when the condensate's mean $k_{x}$ value passes through zero. Our solution of the full time-dependent Gross-Pitaevskii equation for the system therefore shows that accelerating the optical lattice at $-5.0 \mathrm{~ms}^{-2}$ causes the condensate to perform Bloch oscillations in the lowest-energy band of the optical lattice, as observed in the experiments of Morsch et al. [4].

We now compare our full quantum-mechanical simulations with the corresponding semiclassical trajectory of a pointlike particle in the lowest-energy band of the optical lattice. In the optical lattice rest frame, the single-particle trajectories $x(t)$ and $k_{x}(t)$ in real and reciprocal space are determined by the semiclassical equations of motion $d x / d t$ $=\hbar^{-1} d E\left(k_{x}\right) / d k_{x}$ and $d k_{x} / d t=\hbar^{-1} F_{x}$ [32], where $E\left(k_{x}\right)$ is the energy-wave-number dispersion relation for the band and $F_{x}$ is the force along the $x$ axis produced by the acceleration of the optical lattice and by the harmonic trap. The semiclassical trajectories in real and reciprocal space are shown by the open circles in Figs. 2(a) and 2(b), respectively. These paths agree with the corresponding quantum-mechanical calculations to within a few percent. The good agreement between the two theories demonstrates that the condensate behaves like a pointlike particle, obeying semiclassical laws of energy band transport.

As explained in our previous paper [25], at Bragg reflection the condensate wave function is a standing wave. At each maximum in $V_{\mathrm{OL}}(x)$, the atom density is zero and the phase of the condensate wave function changes abruptly by $\pi$. In our earlier work, we showed that this standing wave can generate solitons, which eventually decay into vortex rings. However, in system $A$, the standing wave has no longterm effect on the internal structure of the condensate. Indeed, even though the condensate Bragg reflects five times during our simulation, no solitons are produced, and the $k_{x}$ 
distribution of the atoms remains very narrow [see Fig. 2(b)]. Figures 2(c) and 2(d) show the condensate atom density profile in the $x-r$ plane at the start $(t=0 \mathrm{~ms})$ and end $(t=8 \mathrm{~ms})$ of the simulation, respectively. Comparison of these figures reveals that there is almost no change in the internal structure of the condensate during the motion.

Even though there has been little change in the internal structure of the condensate, the Bloch oscillations are not completely regular in two respects. First, Fig. 2(a) shows a gradual reduction in the amplitude of the Bloch oscillations. Second, the period of the oscillations decreases during the motion. This second effect can be seen most easily in Fig. 2(b), which shows that the condensate's mean $k_{x}$ value rises at an increasing rate throughout the simulation. The decreasing amplitude and period of the Bloch oscillations both originate from the force exerted by the static magnetic trap and, as such, are entirely different from the damping processes that we described in previous work [25]. As the optical lattice drags the condensate towards more negative values of $x$, the trap imparts an additional and increasing force on the condensate, which tends to push it back towards larger values of $x$, that is, back towards the minimum in the trap potential. This positive force due to the trap adds to the inertial force imparted by the accelerating lattice. Consequently, the condensate is subjected to an ever-increasing total force driving it through $k$ space in the positive $k_{x}$ direction. As a result, the period and amplitude of the oscillations both decrease with increasing time. In our previous studies [25], this process was not observed because the condensates were accelerated through a static lattice by displacing the harmonic trap. Note that in the present work, the period and amplitude of the quantum mechanical and the single-particle semiclassical trajectories in system $A$ [Fig. 2(a)] both change with time in the same way. Consequently, these changes must have a fundamentally different origin from the damping mechanism proposed previously [25], which is due to interatomic interactions and collective excitations. Accelerating the condensate further and further from the trap potential minimum will eventually cause the band to break and the Bloch oscillations to break down. This occurs when the product of the lattice period and the total inertial force on the condensate becomes approximately equal to the width of the lowest-energy band. For the parameters described above, this condition is fulfilled when $t=10.5 \mathrm{~ms}$.

We propose that solitons are not produced during the simulation shown in Fig. 2 because the period of the Bloch oscillations is much shorter than those studied in our previous work [25], in which solitons were created by Bragg reflection. The time scale in which the condensate density profile reacts to a perturbation is characterized by the correlation time $t_{c}$, given by the healing length divided by the speed of sound [23]. For the simulation summarized in Fig. 2, the Bloch period $T=2.2 \mathrm{~ms}$ is comparable to $t_{c}=m / 2 \sqrt{2} h n_{M} a$ $\simeq 0.9 \mathrm{~ms}$, where $n_{M}$ is the mean atom density. Consequently, the condensate does not have sufficient time to form solitons in response to the phase and density imprinting associated with Bragg reflection. Hence, soliton production is suppressed, and the internal structure of the atom cloud remains almost unchanged by the motion. Within this picture, soliton production should occur at lower lattice accelerations, corre-
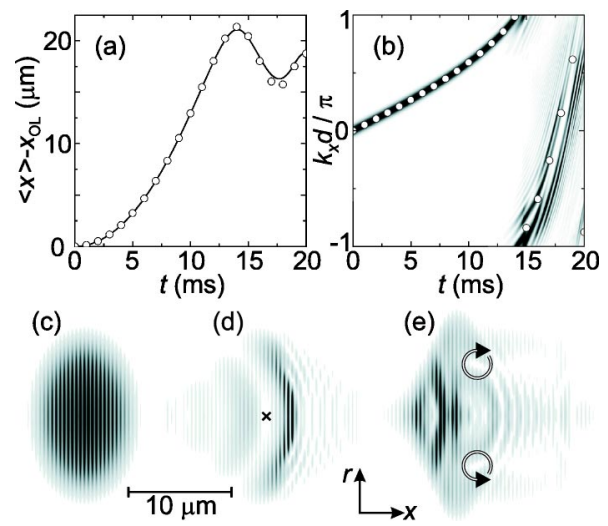

FIG. 3. (a) Solid curve: $\langle x\rangle-x_{\mathrm{OL}}$ vs $t$ calculated for system $A$ with $\bar{a}=-0.3 \mathrm{~ms}^{-2}$. Open circles: corresponding semiclassical trajectory. (b) Gray-scale plot: $\left|f\left(k_{x}, t\right)\right|^{2}$ (white $=0$, black high) for system $A$ with $\bar{a}=-0.3 \mathrm{~ms}^{-2}$. Open circles: corresponding semiclassical trajectory. (c), (d), and (e) Gray-scale plots of condensate density (white $=0$, black high) in the $x-r$ plane (axes inset) for $t$ $=0 \mathrm{~ms}, 16.0 \mathrm{~ms}$, and $18.0 \mathrm{~ms}$, respectively. Plots are symmetrical under rotation about the $x$ axis. Horizontal bar shows scale. Cross in (d) marks center of a soliton. Arrows in (e) show direction of circulation around vortices.

sponding to longer Bloch oscillations, for which $t_{c} \ll T$. To test this hypothesis, we now consider the dynamics of system $A$ for a very small lattice acceleration of $-0.3 \mathrm{~ms}^{-2}$. Semiclassical theory predicts that the resulting Bloch oscillations will have a period of $17.6 \mathrm{~ms}$. Since $t_{c}$ remains $\sim 0.9 \mathrm{~ms}$, the condition $t_{c} \ll T$ is satisfied.

Figure 3(a) shows the time evolution of $\langle x\rangle-x_{\mathrm{OL}}$ for a lattice acceleration of $-0.3 \mathrm{~ms}^{-2}$, and Fig. 3(b) shows the corresponding Fourier power $\left|f\left(k_{x}, t\right)\right|^{2}$. The condensate dynamics are radically different from those shown in Fig. 2 for $\bar{a}=-5.0 \mathrm{~ms}^{-2}$. As $t$ increases from zero, the condensate's mean $k_{x}$ value increases as the atoms accelerate up the first energy band. At $t=14.1 \mathrm{~ms}$, the mean $k_{x}$ value reaches the Brillouin-zone boundary [Fig. 3(b)], and the condensate undergoes Bragg reflection. But, in contrast to the case of high acceleration, after Bragg reflection the Bloch oscillation amplitude decreases dramatically [Fig. 3(a)]. Furthermore, multiple peaks appear in $\left|f\left(k_{x}, t\right)\right|^{2}$, and the $k_{x}$ distribution of the atoms becomes broad, extending across approximately half the Brillouin zone.

As before, we compare our full quantum-mechanical simulations to the corresponding semiclassical trajectory of a pointlike particle in the lowest-energy band of the optical lattice, shown by the open circles in Figs. 3(a) and 3(b). As for $\bar{a}=-5.0 \mathrm{~ms}^{-2}$, the two theories agree to within a few percent. But for $\bar{a}=-0.3 \mathrm{~ms}^{-2}$, the effect of the magnetic trap on the motion is more obvious. First, it causes a severe reduction in the amplitude of the Bloch oscillations. Second, the condensate's mean $k_{x}$ value rises at a rapidly increasing rate throughout the simulation [Fig. 3(b)]. But the principal difference between the two theories is the appearance of multiple peaks in $\left|f\left(k_{x}, t\right)\right|^{2}$ for $t \gtrsim 14.1 \mathrm{~ms}$., which a semiclassical model cannot hope to explain.

This behavior can be understood by considering the internal structure of the condensate, which is shown for various 


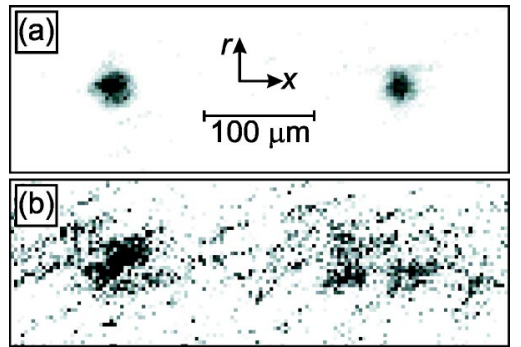

FIG. 4. (a) Experimental gray-scale image (white $=0$, black high) of the condensate in $x-r$ plane (axes inset) for $\bar{a}=-5.0 \mathrm{~ms}^{-2}$. The optical lattice and trap were switched off when $k_{x} \approx \pm \pi / d$, (i.e., at the Brillouin-zone boundary) and the condensate was subsequently allowed to expand freely for $21 \mathrm{~ms}$. Horizontal bar shows scale. (b) Corresponding image of the condensate for $\bar{a}$ $=-0.3 \mathrm{~ms}^{-2}$. [Note that the dynamic range of the gray scale for (b) is only $1 / 3$ of that for (a).]

times in Figs. 3(c)-3(e). As for higher lattice accelerations, a standing wave is formed at the point of Bragg reflection. This standing wave has a density node and an associated $\pi$ phase shift at each maximum in $V_{\mathrm{OL}}(x)$ [25]. Consequently, the density and phase variations induced by Bragg reflection are similar to those realized in recent experiments [21,23,24], in which laser illumination was used to imprint the condensate with density minima and $\pi$ phase shifts, which subsequently evolved into dark solitons. In the same way, the density minima and $\pi$ phase shifts in the standing wave formed at Bragg reflection can evolve into dark solitons, an example of which is marked by the cross in Fig. 3(d). The solitons then decay swiftly via the snake instability $[18,33]$ into vortex rings, which lie in a plane perpendicular to the $x$ axis. Figure 3(e) shows a cross section through such a vortex ring that has formed in the center of the condensate at $t$ $=18.0 \mathrm{~ms}$. The core of the vortex ring cuts the $x$ - $r$ plane at two points, which are enclosed by arrows indicating the direction of circulation. The formation of solitons and vortices disrupts the internal structure of the condensate and broadens the $k_{x}$ distribution of the atom cloud [Fig. 3(b)].

To confirm our theoretical predictions, we conducted an experiment to realize system $A$. When the rubidium atoms had been loaded into the optical lattice [34], we began linearly chirping the frequency offset between the two counterpropagating lattice beams, and so accelerated the optical potential at a constant rate. After a time $t$, we switched off both the optical lattice and the magnetic trap, in which the condensate was held, and observed the resulting interference pattern within the atom cloud after a time of flight of $21 \mathrm{~ms}$.

We now examine results for which the optical and magnetic potentials were switched off when $k_{x} \approx \pi / d$ (i.e., when the condensate first reaches the Brillouin-zone boundary). For a lattice acceleration of $\bar{a}=-5.0 \mathrm{~ms}^{-2}$, we found that there is no disruption of the condensate internal structure during Bragg reflection. Figure 4(a) shows an image of the atom cloud following the period of free expansion. Note that the condensate has split into two smaller atom clouds corresponding to the two distinct wave-number components $k_{x}$ $= \pm \pi / d$ in the original condensate at Bragg reflection. Both condensates have tightly peaked density profiles, indicating

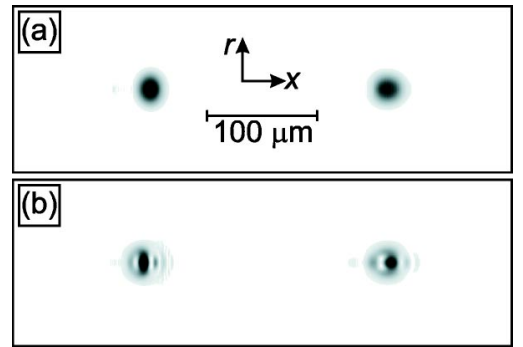

FIG. 5. (a) Theoretical gray-scale image of the condensate density (white $=0$, black high) in $x-r$ plane (axes inset) for $\bar{a}$ $=-5.0 \mathrm{~ms}^{-2}$. The optical lattice and trap were switched off when $k_{x} \approx \pm \pi / d$, (i.e., at the Brillouin-zone boundary) and the condensate was subsequently allowed to expand freely for $21 \mathrm{~ms}$. Horizontal bar shows scale. (b) Corresponding theoretical gray-scale plot of the condensate density for $\bar{a}=-0.3 \mathrm{~ms}^{-2}$.

that there have been no significant changes in the internal structure of the atom cloud, and that the condensate has moved through the energy band like a pointlike semiclassical particle. By contrast, following an acceleration of $\bar{a}$ $=-0.3 \mathrm{~ms}^{-2}$, each of the two subcondensates has a diffuse and fragmented density profile [Fig. 4(b)], indicating that the internal structure of the condensate has changed radically during the motion.

To relate the experimental results directly to the theory, we performed simulations of the condensate evolution, including the period of free expansion, using explicit experimental parameters. The simulations for $\bar{a}=-5.0 \mathrm{~ms}^{-2}$ and $\bar{a}$ $=-0.3 \mathrm{~ms}^{-2}$ presented in Fig. 5(a) and 5(b), respectively, are equivalent to the experimental images in Figs. 4(a) and 4(b). Figure 5(a) shows two tightly peaked condensates that have smooth atom density profiles, in good agreement with the experimental observations shown in Fig. 4(a). In contrast, Fig. 5(b) shows two irregularly shaped condensates that have complicated internal structures. This is in reasonable qualitative agreement with the diffuse and fragmented atom cloud observed in experiment [Fig. 4(b)]. However, the changes in the atom density profile are more pronounced in the experiment [Fig. 4(b)] than in the corresponding simulation [Fig. $5(\mathrm{~b})$ ]. This is probably due to heating of the atom cloud, which cannot be described by the Gross-Pitaevskii equation.

The results presented in this section demonstrate that slow Bloch oscillations $\left(t_{c} \ll T\right)$ promote the production of solitons and vortex rings, and, conversely, that fast Bloch oscillations $\left(t_{c} \approx T\right)$ suppress this excitation process. This conclusion is supported by the theoretical work of Holthaus [9], who, using a one-dimensional calculation, demonstrated a broadening of the condensate and distortion of the Bloch oscillations under weak forcing through an optical lattice. Similar ideas were discussed by Konotop and Salerno [10], and $\mathrm{Wu}$ and Niu [11], who reported that Bose-Einstein condensates in Bloch states near the Brillouin-zone boundary are unstable with respect to small perturbations.

\section{EXPLOSIVE EXPANSION OF SYSTEM $B$}

The parameters of system $B$ are $N=3 \times 10^{5}, \quad V_{0}$ $=23.3 \mathrm{peV}=1.55 E_{R}, d=397.5 \mathrm{~nm}, \omega_{x}=2 \pi \times 8.7 \mathrm{rad} \mathrm{s}^{-1}$, and 
(a)

(b)

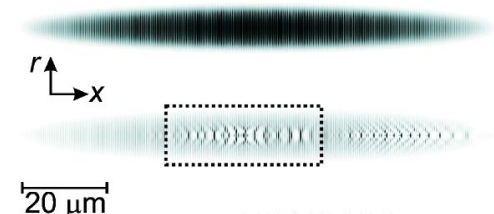

(c)

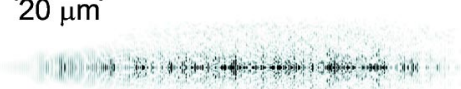

FIG. 6. Gray-scale plots of density (white $=0$, black high) in the $x-r$ plane (axes inset) for system $B$ with $\Delta x=150 \mu \mathrm{m}$ and $t=0 \mathrm{~ms}$ (a), $14.0 \mathrm{~ms}$ (b), and $18.0 \mathrm{~ms}$ (c). Plots are symmetrical under rotation about the $x$ axis. Horizontal bar shows scale. Region within dashed box in (b) is shown enlarged in Fig. 8(d).

$\omega_{r}=2 \pi \times 90 \mathrm{rad} \mathrm{s}^{-1}$. These parameters create a cigar-shaped condensate, with a peak atom density of $\sim 1.7 \times 10^{14} \mathrm{~cm}^{-3}$, which is approximately five times larger than the corresponding density for system $A$. The width of the lowest-energy band of the optical lattice is $10.0 \mathrm{peV}=0.67 E_{R}$, which is slightly larger than that of system $A$. Figure 6(a) shows the equilibrium ground-state atom density profile calculated for the combined trap and optical potential in system $B$.

In contrast to system $A$, the optical lattice in system $B$ is not accelerated. Instead, as in the experiments of Ref. [8], the condensate is set in motion by suddenly displacing the harmonic trap through a distance $\Delta x$ along the $x$ axis at time $t$ $=0$. Immediately after the trap displacement, the potential energy of each atom in the condensate increases by

$$
\Delta V \simeq \frac{1}{2} m \omega_{x}^{2}(\Delta x)^{2} .
$$

This potential energy is then converted into kinetic energy as the condensate accelerates up the first energy band.

We now consider the condensate dynamics produced by a trap displacement $\Delta x=150 \mu \mathrm{m}$, which was realized in the experiments of Ref. [8]. For this displacement, the semiclassical model of single-particle band transport predicts that the resulting Bloch oscillations will have a period of $\sim 28 \mathrm{~ms}$. For system $B, t_{c} \simeq 0.2 \mathrm{~ms}$, so the soliton formation condition $t_{c} \ll T$, proposed in Sec. III, is satisfied. Hence, a large trap displacement of $150 \mu \mathrm{m}$ might be expected to induce rapid generation of solitons and vortices and, consequently, a breakdown of the Bloch oscillations.

Furthermore, the high density of the condensate in system $B$ might also be expected to promote soliton and vortex production. To explain the reason for this, let us first consider the condensate in system $A$ undergoing Bloch oscillations. In this system, at the first Bragg reflection, the local mean atom density is $\sim 3 \times 10^{13} \mathrm{~cm}^{-3}$ near the center of the condensate. Hence, the local healing length $\xi=1 / \sqrt{8 \pi n_{M} a} \simeq 0.5 \mu \mathrm{m}$ $\simeq 1.25 d$, which corresponds to a soliton width $w \simeq 2 \xi$ $\simeq 2.5 d$ [24]. Since this width is much greater than the separation of adjacent density minima and $\pi$ phase slips imprinted at the point of Bragg reflection, it is difficult for these features to evolve into solitons. But, some solitons do, nevertheless, form in system $A$ if the acceleration is slow enough [see Fig. 3(d)].

By contrast, solitons should form more readily if $w \leq d$ [25], because the standing wave then more closely resembles

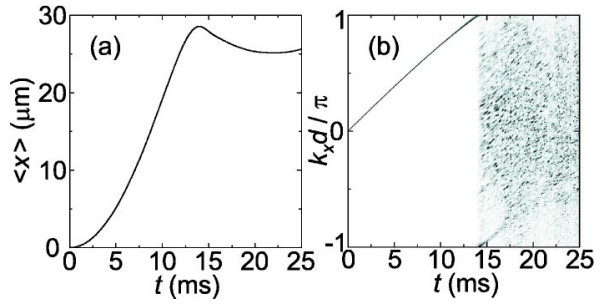

FIG. 7. (a) $\langle x\rangle$ vs $t$ calculated for system $B$ with $\Delta x=150 \mu \mathrm{m}$. (b) Corresponding gray-scale plot of $\left|f\left(k_{x}, t\right)\right|^{2}$ (white $=0$, black high).

a chain of black solitons. This condition can be satisfied in a high-density condensate, which has a small healing length and, consequently, a small soliton width $w$. In the case of system $B$, the density is high enough for the soliton width to match closely the separation of adjacent density minima and $\pi$ phase slips in the standing wave formed at Bragg reflection. Hence, the system might be expected to exhibit rapid soliton and vortex production.

Figures 7(a) and 7(b) show the time evolution of $\langle x\rangle$ and $\left|f\left(k_{x}, t\right)\right|^{2}$ in system $B$ for a trap displacement of $150 \mu \mathrm{m}$, calculated using Eqs. (7) and (11) (note that $\bar{a}=0$ for system $B$ ). Figure 7(b) shows that the mean $k_{x}$ value of the condensate reaches the Brillouin-zone boundary at approximately $13 \mathrm{~ms}$. As for system $A$, at the point of Bragg reflection a standing wave forms, which has nodal planes and associated $\pi$ phase shifts at each maximum in $V_{\mathrm{OL}}(x)$. But, for system $B$, the local mean atom density at Bragg reflection is $\sim 1.6$ $\times 10^{14} \mathrm{~cm}^{-3}$ near the center of the condensate. Consequently, the local healing length is approximately $0.2 \mu \mathrm{m} \simeq 0.5 d$, which corresponds to a soliton width $w \simeq 2 \xi \simeq d$. Hence, for these parameters, both the condition $w \leqq d$ and the condition $t_{c} \ll T$ are satisfied. As a result, the standing wave formed on Bragg reflection should evolve rapidly into a chain of stationary solitons.

As expected from this argument, Bragg reflection does indeed cause the self-assembly of $\sim 20$ stationary solitons, which form a chain across the central third of the condensate. Figure 6(b) shows the compact cigar-shaped density profile of the condensate just after the first Bragg reflection. Each extended white area within the dashed box contains several tightly grouped solitons. The region within the dashed box is shown enlarged in Fig. 8(d), which reveals $\sim 10$ stationary solitons.

Figure 8 shows key stages in the formation of the solitons, and their subsequent decay into vortex rings. Figure 8(a) shows the atom density profile in the central third of the condensate immediately after the first Bragg reflection. The nodal planes in the standing wave appear as vertical white stripes. As time increases, depressions grow in the condensate [Figs. 8(b) and 8(c)], which, after $14 \mathrm{~ms}$, have evolved into groups of solitons (large white regions in Fig. 8(d)], which form a chain along the $x$ axis. It is actually difficult to display the soliton chain in a single image because the solitons decay extremely rapidly into vortex rings. In Fig. 8(d), some of the solitons have already begun to break up, while others are still forming. Since their decay is so rapid, the solitons create a chain of vortex rings [seen most clearly in 


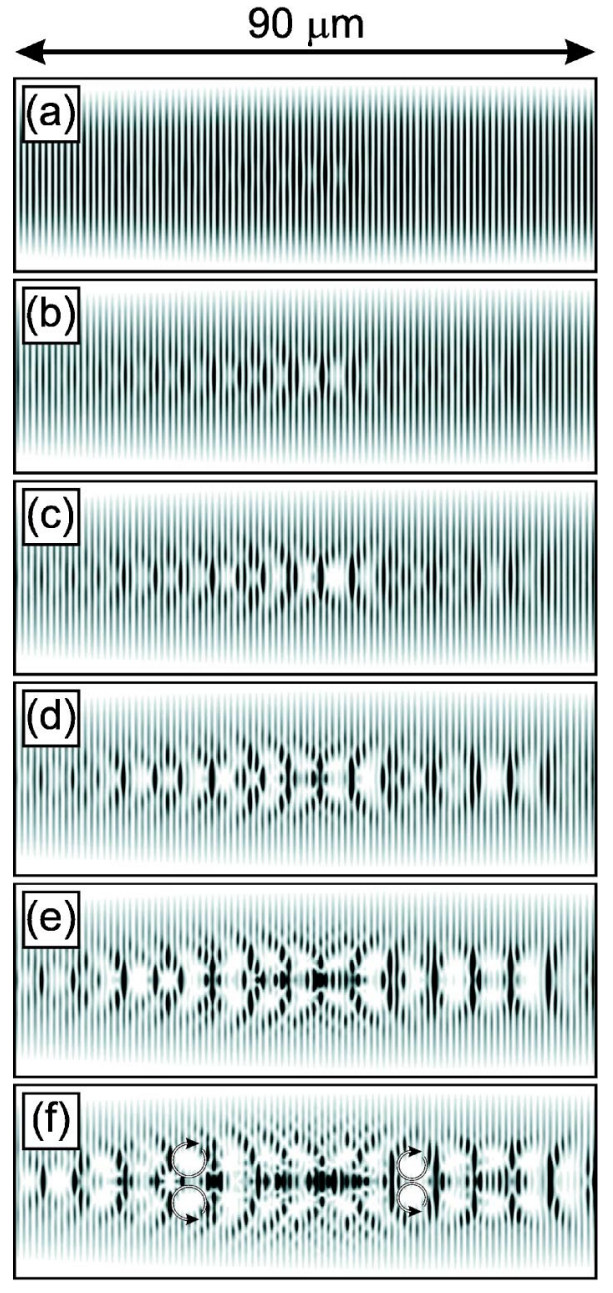

FIG. 8. Theoretical gray-scale plots of atom density profile (white $=0$, black high) in the $x-r$ plane in central third of the condensate for system $B$ with $\Delta x=150 \mu \mathrm{m}$ and $t=13.4 \mathrm{~ms}$ (a), $13.6 \mathrm{~ms}$ (b), $13.8 \mathrm{~ms}$ (c), $14.0 \mathrm{~ms}$ (d), $14.2 \mathrm{~ms}$ (e), and $14.4 \mathrm{~ms}$ (f). (d) corresponds to the region within the dashed box in Fig. 6(b). Arrows in (f) show direction of circulation around two vortex rings. Horizontal arrow at the top of figure shows scale.

Figs. 8(e) and 8(f)], which form a complex interacting system. The cores of two of the vortex rings are enclosed by arrows in Fig. 8(f), which indicate the direction of circulation.

The interactions now create a large internal strain, which causes the condensate to explode laterally (perpendicular to the $x$ axis), resulting in the diffuse and fragmented atom density profile shown in Fig. 6(c). The explosion has a dra- matic effect on the $k_{x}$ distribution of the atoms [Fig. 7(b)], which is initially extremely narrow but, at the point of Bragg reflection, spreads through the whole Brillouin zone. The condensate loses all coherence in $k$ space, and so does not perform Bloch oscillations. Instead, once the condensate has reached the Brillouin-zone boundary, its center of mass remains approximately stationary [see Fig. 7(a)]. This behavior is consistent with the experimental observations of Burger et al. [8], who noted that, following a trap displacement of $150 \mu \mathrm{m}$, the atom cloud broadens and distorts and the center-of-mass motion is severely damped.

\section{CONCLUSION}

We have presented experimental and theoretical studies of the transport and disruption of Bose-Einstein condensates in optical lattices. Our experimental results are in good quantitative agreement with the corresponding simulations, performed by solving the three-dimensional time-dependent Gross-Pitaevskii equation for explicit experimental parameters. Our simulations also account for the complex condensate dynamics observed in previous experiments by Burger and co-workers [8], and provide fresh insights for understanding these experiments. Moreover, they enable us to identify parameter regimes in which qualitatively new physical effects should be observable, in particular the dramatic explosion induced by the Bragg reflection of high-density condensates.

Our study of two different condensate systems provides a broad picture of the nature of condensate transport in optical lattices. Initially, we considered a set of parameters for which the condensate undergoes approximately periodic Bloch motion, and its internal structure hardly changes with $t$, as in recent experiments [4]. But in other parameter regimes, the center-of-mass motion disrupts the condensate through the production of solitons and vortices. We have shown that solitons and vortices form most readily in high-density condensates undergoing slow Bloch oscillations, such that $w \lesssim d$ and $t_{c} \ll T$. New experiments are now needed to explore the rich dynamical properties of condensates in optical lattices and, in particular, to determine whether the catastrophic effect of soliton and vortex formation on high-density condensates, predicted by our calculations, occur in actual systems.

\section{ACKNOWLEDGMENTS}

We would like to express our gratitude to M. Anderlini, D. Ciampini, E. Courtade, and J. H. Müller for their help in acquiring the experimental data. This work was supported by EPSRC UK.
[1] P. L. Gould, G. A. Ruff, and D. E. Pritchard, Phys. Rev. Lett. 56, 827 (1986).

[2] M. B. Dahan, E. Peik, J. Reichel, Y. Castin, and C. Salomon, Phys. Rev. Lett. 76, 4508 (1996).

[3] S. R. Wilkinson, C. F. Bharucha, K. W. Madison, Q. Niu, and M. G. Raizen, Phys. Rev. Lett. 76, 4512 (1996).
[4] O. Morsch, J. H. Müller, M. Cristiani, D. Ciampini, and E. Arimondo, Phys. Rev. Lett. 87, 140402 (2001).

[5] B. P. Anderson and M. A. Kasevich, Science 282, 1686 (1998).

[6] M. Kozuma, L. Deng, E. W. Hagley, J. Wen, R. Lutwak, K. Helmerson, S. L. Rolston, and W. D. Phillips, Phys. Rev. Lett. 
82, 871 (1999).

[7] U. V. Poulsen and K. Mølmer, Phys. Rev. A 65, 033613 (2002).

[8] S. Burger, F. S. Cataliotti, C. Fort, F. Minardi, M. Inguscio, M. L. Chiofalo, and M. P. Tosi, Phys. Rev. Lett. 86, 4447 (2001).

[9] M. Holthaus, J. Opt. B: Quantum Semiclassical Opt. 2, 589 (2000).

[10] V. V. Konotop and M. Salerno, Phys. Rev. A 65, 021602 (2002).

[11] B. Wu and Q. Niu, Phys. Rev. A 64, 061603 (2001).

[12] A. Trombettoni and A. Smerzi, Phys. Rev. Lett. 86, 2353 (2001).

[13] D.-I. Choi and Q. Niu, Phys. Rev. Lett. 82, 2022 (1999).

[14] K. Berg-Sørensen and K. Mølmer, Phys. Rev. A 58, 1480 (1998).

[15] K. P. Marzlin and W. Zhang, Phys. Rev. A 59, 2982 (1999).

[16] F. S. Cataliotti, S. Burger, C. Fort, P. Maddaloni, F. Minardi, A. Trombettoni, A. Smerzi, and M. Inguscio, Science 293, 843 (2001).

[17] J. C. Bronski, L. D. Carr, B. Deconinck, and J. N. Kutz, Phys. Rev. Lett. 86, 1402 (2001).

[18] Z. Dutton, M. Budde, C. Slowe, and L. V. Hau, Science 293, 663 (2001).

[19] M. Greiner, I. Bloch, O. Mandel, T. W. Hänsch, and T. Esslinger, Phys. Rev. Lett. 87, 160405 (2001).

[20] M. Greiner, O. Mandel, T. Esslinger, T. W. Hänsch, and I. Bloch, Nature (London) 415, 39 (2002).

[21] S. Burger, L. D. Carr, P. Öhberg, K. Sengstock, and A. Sanpera, Phys. Rev. A 65, 043611 (2002).
[22] M. L. Chiofalo and M. P. Tosi, Phys. Lett. A 268, 406 (2000).

[23] S. Burger, K. Bongs, S. Dettmers, W. Ertmer, K. Sengstock, A. Sanpera, G. V. Shlyapnikov, and M. Lewenstein, Phys. Rev. Lett. 83, 5198 (1999).

[24] J. Denschlag et al., Science 287, 97 (2000).

[25] R. G. Scott, A. M. Martin, T. M. Fromhold, S. Bujkiewicz, F. W. Sheard, and M. Leadbeater, Phys. Rev. Lett. 90, 110404 (2003).

[26] B. Wu and Q. Niu, Phys. Rev. Lett. 89, 088901 (2002).

[27] S. Burger, F. S. Cataliotti, C. Fort, F. Minardi, M. Inguscio, M. L. Chiofalo, and M. P. Tosi, Phys. Rev. Lett. 89, 088902 (2002).

[28] M. Abramowitz and I. A. Stegun, Handbook of Mathematical Functions (Dover, New York, 1965).

[29] R. G. Scott, S. Bujkiewicz, T. M. Fromhold, P. B. Wilkinson, and F. W. Sheard, Phys. Rev. A 66, 023407 (2002).

[30] E. A. Burt, R. W. Ghrist, C. J. Myatt, M. J. Holland, E. A. Cornell, and C. E. Wieman, Phys. Rev. Lett. 79, 337 (1997).

[31] W. H. Press, S. A. Teukolsky, W. T. Vetterling, and B. P. Flannery, Numerical Recipes, the Art of Scientific Computing (Cambridge University Press, Cambridge, 1994).

[32] N. W. Ashcroft and N. D. Mermin, Solid State Physics (Saunders, Philadelphia, 1976).

[33] B. P. Anderson, P. C. Halijan, C. A. Regal, D. L. Feder, L. A. Collins, C. W. Clark, and E. A. Cornell, Phys. Rev. Lett. 86, 2926 (2001).

[34] M. Cristiani, O. Morsch, J. H. Müller, D. Ciampini, and E. Arimondo, Phys. Rev. A 65, 063612 (2002). 